\title{
Hybrid approach for the assessment of vibrations and re-radiated noise in buildings due to railway traffic: Concept and preliminary validation
}

\author{
P. Alves Costa, R. Arcos, P. Soares \& A. Colaço \\ CONSTRUCT - Institute of R\&D in Structures and Construction, Faculty of Engineering, University of Porto, Porto, \\ Portugal
}

\begin{abstract}
In this paper, a hybrid experimental/numerical methodology for the assessment of railway induced ground-borne noise and vibration in buildings based on experimental measurement in the ground surface is proposed and validated using synthetic data. The application of such approach is focused in the prediction of vibrations and re-radiated noise inside buildings to be constructed nearby existing railway infrastructures. After the presentation of the concept, the numerical validation of the proposed approach is shown for a $2 \mathrm{D}$ example. The proposed method can be easily generalized for 3D conditions.
\end{abstract}

\section{INTRODUCTION}

The relevance of environmental problems regarded with vibrations induced by railway subsurface traffic has been growing during the last years. Simultaneously, it can also be noticed that the technical and scientific communities have allocated a considerable effort on the topic, allowing the development of several studies and models for the prediction of the vibrations induced by transportation infrastructures.

One of the most common situations in where a railway-induced noise and vibration assessment is required is the construction of a new building nearby to an existing and operational urban railway line. In such cases, it is recommendable to make a prediction of the re-radiated noise and vibration levels in order to check if mitigation measures must be applied in order to fulfil the standard requirements.

The problem stated above has been addressed following distinct approaches: i) empirical; ii) analytical; iii) numerical. Empirical methods, such those proposed by Federal Transportation Administration [1] are useful for scoping stage, nevertheless they are difficult to apply in a detailed approach where the specific properties of the site must be taken into account [2]. On the other hand, analytical models are usually devoted for the prediction of vibrations in free-field and their application is limited for simple geometries [3]. Therefore, numerical approaches can be faced as the method of choice when versatility is a requirement, allowing the inclusion of whole system, from the source to the building, including re-radiated noise prediction $[4,5]$

In the scope of numerical approach, Fiala et al. [6] used a decoupled approach to assess the vibration response of the building. This approach considers a weak coupling between the tunnel/soil and the building/soil systems. For the tunnel/soil system, a two-anda-half-dimensional (2.5D) FEM model for the tunnel and a $2.5 \mathrm{D}$ boundary element method (BEM) model for the soil are used. In contrast, for the building/soil model, a 3D FEM-BEM model is considered. A similar approach was presented by Lopes et al. [7, 8], where, in this case, the soil is modelled by using a $2.5 \mathrm{D}$ FEM with perfect matched layers (PML) as a sub-model of a comprehensive track/tunnel/soil model. As can be seen, several numerical approaches are nowadays available, some of them quite powerful and versatile. However, the usage of such kind of models usually demands reliable input data that is not usually easy to obtain.

Among the different sources of uncertainty, the imperfect knowledge of the local subsoil conditions is found to be a significant source of uncertainty. As explained by Lopes et al. [8] and Papadopoulos et al. [9], the soil condition significantly influences the response of the building. Also the train-track interaction mechanisms are difficult to quantify.

In an attempt to reduce the uncertainty of computational models, hybrid modelling based on the combination between in situ experimental data and a theoretical model is an interesting alternative. In framework of hybrid modelling for soil/building dynamic interaction problems, Auersch [10] presented a semi-empirical model that combines pre-calculated results obtained from detailed numerical models, a database of experimental data build from many experimental measurements and several specific analytical models. Sanayei et al. [11] proposed a hybrid approach where building is numerically modelled and the incident wave field is represented by previously known column base forces or measured vibrations at the loading dock floor.

More recently, Lopez-Mendoza et al. [12] have presented a computationally efficient model to predict the ground-borne railway-induced vibration levels in buildings considering soil-structure interaction. 
In this paper, a new methodology for the prediction of the ground-borne vibration induced by operational urban railway lines in buildings to be constructed in the surroundings of the infrastructure is presented. This method uses railway-induced vibrations measured in the ground where the new building will be constructed to compute a set of virtual forces that represent the incident wave field induced by the nearby operational railway line. These virtual forces are then used on a model of the particular building/soil system to be studied in order to predict the vibration levels at any point of the structure. Thus, this method considers a weak coupling between the railway infrastructure and the building structure. Model is also extended to the prediction of re-radiated noise, following the approach proposed by Colaço et al $[4,13]$ and more recently by Ghangale et al. [5].

\section{APPROACH DESCRIPTION}

\subsection{Generalities}

In this section, the new hybrid methodology for the prediction of the railway-induced ground-borne vibration and noise in buildings to be constructed nearby to railway urban lines is presented. The approach is spited into 4 steps.

\subsection{Step 1}

To apply this approach, it is necessary to record vibrations at the ground surface, in the locations where building foundations will be constructed (collocation points), due to the railway infrastructure operation (see Figure 1). This is the "experimental" step of the approach. Since the methodology is based on frequency domain, time records need to be transformed to the frequency domain.

\subsection{Step 2}

The method is based an approach similar to the method of fundamental solutions (MFS), where it is assumed that a previously known boundary conditions, defined by the so-called collocation points, is approximated by the linear superposition of the effects induced by virtual sources, as depicted in Figure 2. Here, the MFS approach presented by Arcos et al. [14] is applied. In

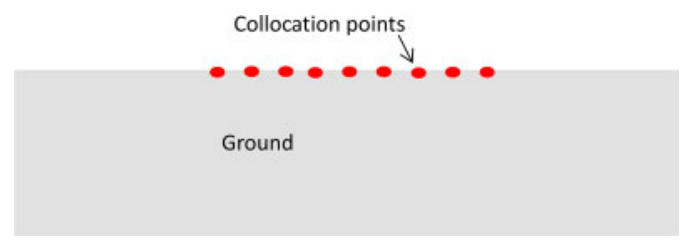

Figure 1. Measurement of vibrations at ground surface close to the location of the foundations of future building. that methodology, the known condition in the collocation points is the measured response in the frequency domain. Although not required, in the present approach it is assumed that the number of virtual sources is equal to the number of collocation points.

The virtual forces, $F_{v}$, are then computed from the known of the displacements at the colocation points (experimental data), $U_{c}$ :

$$
F_{v}=H_{c f}^{-1} U_{c}
$$

where $H_{c f}$ is a square matrix that relates the virtual forces and the collocation points response. This matrix is obtained from a local elastodynamic model, and the solution can be achieved from the application of different numerical approaches such as the finite elements method of the method of fundamental solutions. If the ground can be assumed as homogeneous, the application of MFS can be very attractive [15]. The mathematical details about the procedure to obtain this matrix can be found in $[7,15]$.

\section{$2.4 \quad$ Step 3}

The solution of linear system of equations 1 allows obtaining virtual sources that, in a local model, give rise to a wave field at the collocation points similar to the dynamic response measured in the field. Next step is to construct a numerical model that comprises not only the local ground simulation, but also the building that will be constructed (Figure 3). This model is then subjected to the virtual forces computed in the previous step and the dynamic response of the building is obtained. Mathematically, this step can be expressed as:

$U_{b}=H_{b f} F_{v}$

where $U_{b}$ represents the response of a set of evaluation points placed in the building/soil model and $H_{b f}$ is the receptance matrix that relates the virtual forces and the evaluation points response.

Since the computation of matrix $H b f$ requires a model able to simulate not only the ground but also the building structure, it is interesting to combine different numerical methods in order to achieve accuracy and versatility $[5,15,16]$., or, alternatively, to use FEM-PML approach [4].

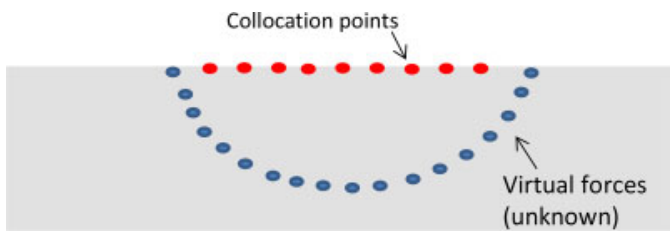

Figure 2. Schematic representation of the location of the location of the collocation points and virtual sources. 


\subsection{Step 4}

The last step comprises the re-radiated noise computation. This can be easily predicted by solving the elasto-acoustic problem, taking into account the velocity of vibration of the walls and slabs that delimit the enclosure acoustic medium. Colaço et al. [4] shown that a weak coupling between elastic domain (building structure) and acoustic medium can be adopted without introducing significant solution error. Following an acoustic MFS approach the boundary condition is defined by the particle velocities in the normal direction to the walls and slabs that delimit the enclosure (collocation points). Therefore, the acoustic pressure inside the enclosure is given by:

$p\left(x, k_{a}\right)=\sum^{N S} A_{m} G\left(x, x_{0}^{(m)}, k_{a}\right)$

In this equation, $G$ corresponds to the Green's function of the sound pressure, $A_{m}$ is the amplitude of the virtual sources located outside of the domain under analysis, as depicted in Figure 4, and NS is the number

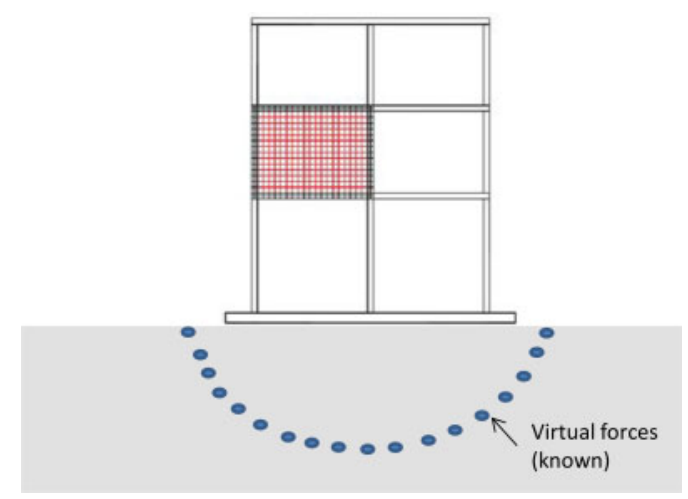

Figure 3. Schematic representation of step 3.

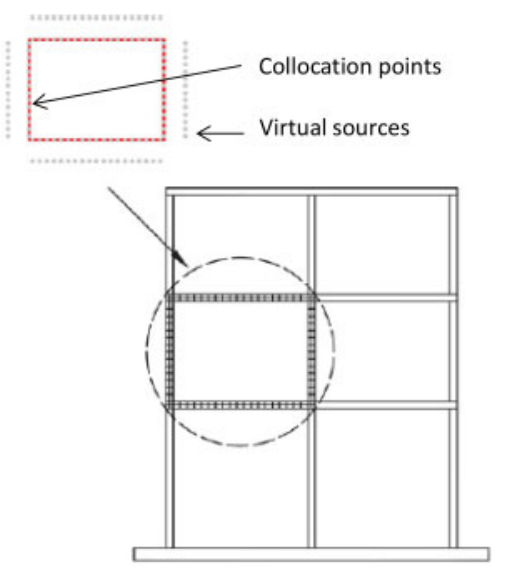

Figure 4. Schematic representation of an MFS model for the acoustic domain $\Omega$. of virtual sources (assumed the same as the number of collocation points).

The amplitude of the virtual sources is obtained from the imposition of the boundary conditions in the set of collocation points. Assuming compatibility of particle velocities along the interface between the elastic and acoustic domain, the virtual sources amplitudes are given the solution of following restriction:

$\sum_{m=1}^{N S}\left[A_{m} H\left(x_{P}^{(i)}, x_{0}^{(m)}, k_{a}, \vec{n}\right)\right]=v_{n, i}$

where $H$ is the Green's function of the particle's velocity.

\section{NUMERICAL VALIDATION OF THE APPROACH}

\subsection{Case study description}

In this section, the previously explained hybrid method is validated numerically. In order to achieve this validation, 2.5D FEM-PML models have been created, based on the approach presented by Lopes et al. [7]. In what concerns to re-radiated noise prediction, an acoustic MFS model, formulated in 2D, was adopted, following the approach suggested by Colaço et al. [4].

For this simple example, the experimental data are replaced by synthetic data generated using a $2.5 \mathrm{D}$ FEM-PML model. This system aims to represent the existing tunnel embedded in a homogenous ground. The tunnel has $8.5 \mathrm{~m}$ of inner diameter, with a tunnel wall thickness of $0.35 \mathrm{~m}$. The center of the tunnel is located at $19.2 \mathrm{~m}$ depth from the ground surface. The mesh created for the tunnel/soil system is presented in Figure 5, where 20 collocation points are uniformly distributed from $14.325 \mathrm{~m}$ to $28.325 \mathrm{~m}$, horizontally from the tunnel center. In a real case, this colocation points correspond to the locations where the measurements of vibrations of the ground surface due to train

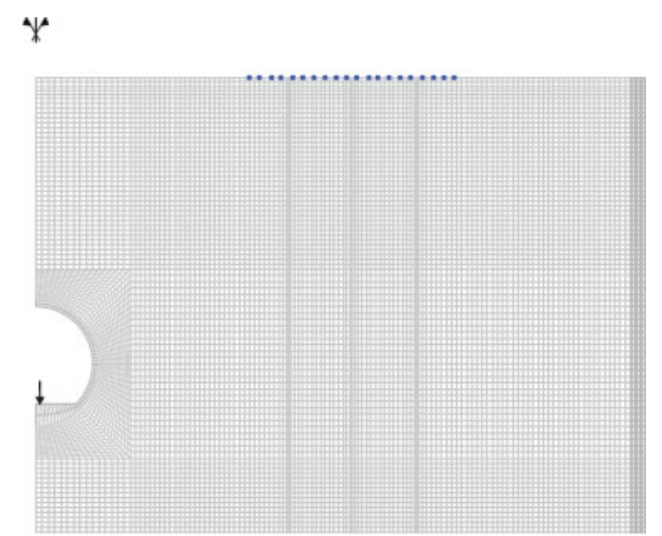

Figure 5. Tunnel-soil system 2.5D FEM-PML mesh and location of the measurement points (collocation points). 
Table 1. Elastodynamic properties adopted for the different materials.

\begin{tabular}{lllll}
\hline Material & $E(\mathrm{GPa})$ & $\rho\left(\mathrm{kg} / \mathrm{m}^{3}\right)$ & $v$ & $\xi$ \\
\hline Soil & 0.39 & 2000 & 0.3 & 0.04 \\
Tunnel & 30 & 2500 & 0.2 & 0.01 \\
Building & 30 & 2500 & 0.2 & 0.01
\end{tabular}

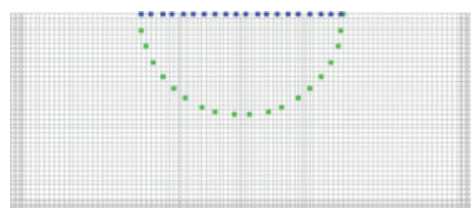

Figure 6. 2.5D FEM-PML mesh of the soil surrounding the future building and location of the measurement points (collocation points) and virtual sources.

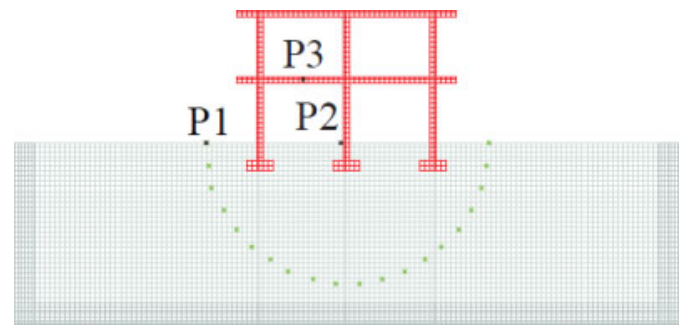

Figure 7. Mesh of the building/soil system, where the position of the evaluation points $\mathrm{P} 1, \mathrm{P} 2$ and $\mathrm{P} 3$ is represented by black points and the position of the virtual forces is highlighted in green.

passage should be measured. Instead of the passage of a train, the excitation assumed in this model consists on a vertical unitary Ricker pulse applied on the tunnel invert. The elastodynamic properties adopted for the case study are depicted in Table 1 .

As previously explained, the development of the step 2 comprises the construction of a numerical or analytical model to compute the terms of matrix $H_{c f}$. In the present study, 2.5D FEM-PML approach was selected to develop this step, being the corresponding 2.5D FEM-PML mesh illustrated in Figure 6. Green dots represented in the figure correspond to the location of the virtual sources, while blue dots correspond to collocation points. The virtual forces, with 20 different locations, are equally spaced along a semi-circumference with $7 \mathrm{~m}$ of radius.

A 3rd numerical model needs to be constructed, now considering the presence of the building, as depicted in Figure 7. This is made up of 2 floors with $3 \mathrm{~m}$ of height and a span between walls of $4 \mathrm{~m}$. The thickness of the walls and the floors is considered to be $0.3 \mathrm{~m}$. The depth of the shallow foundations is $1.4 \mathrm{~m}$ from the ground surface. From this model, the Green's functions matrix $H_{b f}$ that relates the response on the evaluation

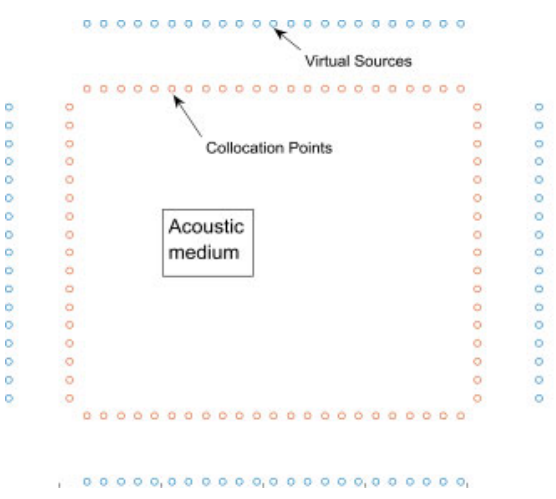

Figure 8. Collocation points and virtual sources location for the computation of re-radiated noise in the upper right enclosure of the building.

points P1, P2 and P3 with the virtual forces can be obtained. Then, the response on the evaluation points can be obtained by using equation 2 .

In the cases where prediction of re-radiated noise is also an objective, this can be done by computing the particle velocity in the direction normal to walls and slabs that delimit the enclosure. As previously presented, this information is used as boundary conditions to solve the acoustic problem, which can be done by MFS approach through equations 3 and 4 . In the present case, the noise is only assessed in the upper right enclosure of the building, which was modeled by the MFS using the collocation points and virtual sources depicted in Figure 8.

With the aim of checking the presented methodology, a model of the corresponding comprehensive tunnel/soil/building system has been created. The mesh of this model is presented in Figure 9, where the virtual sources are only highlighted to show the correspondence with the hybrid model. In order to be in accordance with the models used for the hybrid methodology, the center of the building is located at $21.35 \mathrm{~m}$ horizontally from the center of the tunnel. In this model, the excitation considered is also a vertical unitary force applied on the tunnel invert.

The response obtained by this reference model in any evaluation point of the building and the surrounding ground should match the response in the same points obtained by the hybrid methodology. The comparison between the responses of both models is presented in the following sub-section.

\section{$3.22 D$ validation}

In this section, a comparison of the response obtained by the reference and hybrid models for the $2 \mathrm{D}$ problem is shown. The results for a $2 \mathrm{D}$ case are obtained from a $2.5 \mathrm{D}$ modelling by considering the wavenumber equal to zero. Figure 10 and Figure 11 show this comparison for the evaluation points $\mathrm{P} 1$ and $\mathrm{P} 2$, respectively, for the vertical displacement in time and frequency domains. As shown, both responses are in good agreement up to $200 \mathrm{~Hz}$. For higher frequencies, both models should 


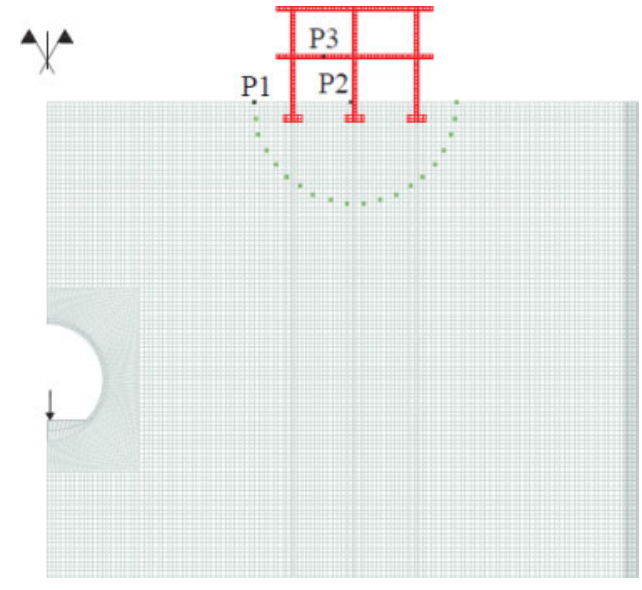

Figure 9. Mesh of comprehensive tunnel/soil/building system, where the position of the evaluation points P1, P2 and $\mathrm{P} 3$ is represented by black points and the position of the collocation points used in the hybrid method is highlighted in blue.
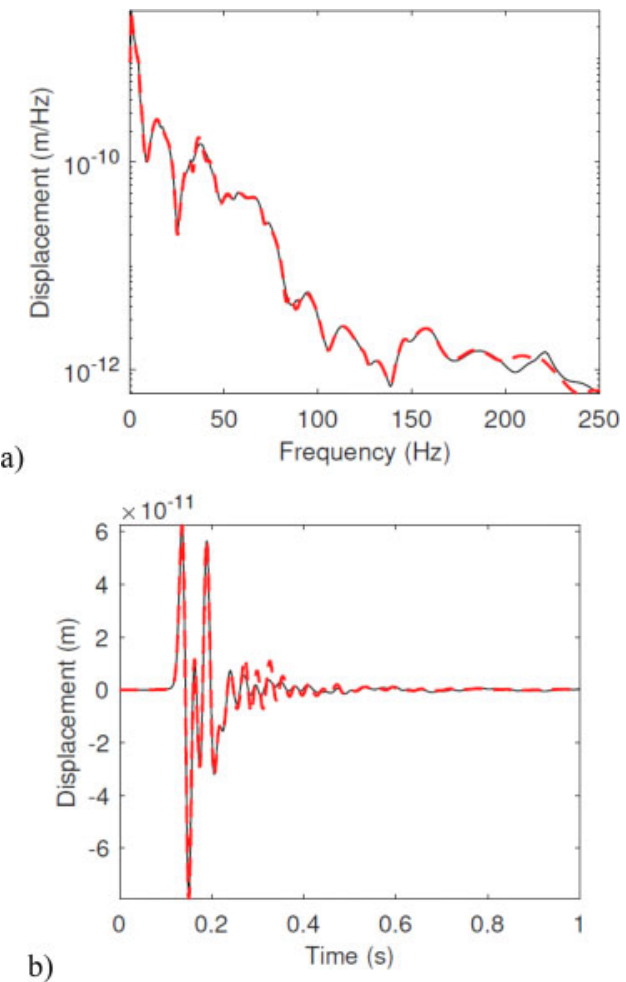

Figure 10. Vertical displacement of point P1: a) frequency domain; b) time domain. (Continuous black lines represent the results of the reference model and the dashed red lines the results of the hybrid method).

not behave properly since the elements size of finite elements is not small enough.

In terms of re-radiated noise, Figure 12 shows the evolution of noise pressure with frequency predicted

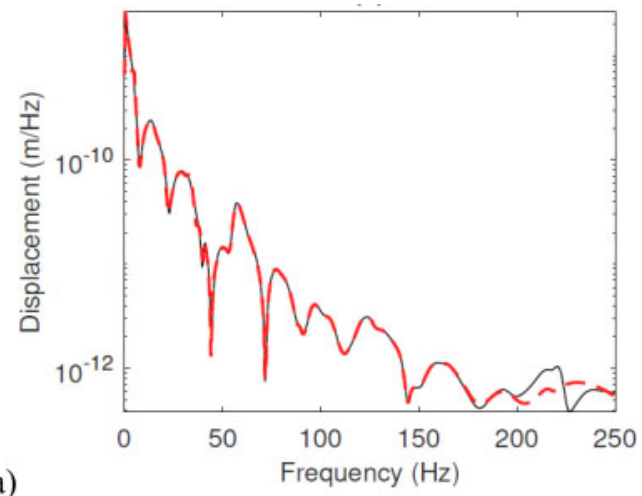

a)

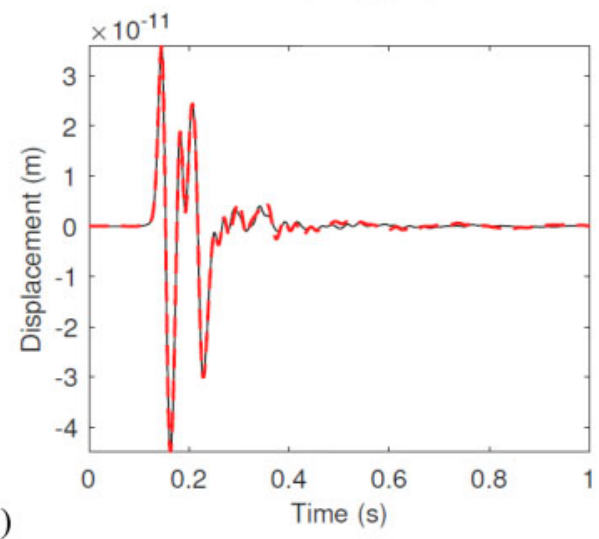

Figure 11. Vertical displacement of point P1: a) frequency domain; b) time domain. (Continuous black lines represent the results of the reference model and the dashed red lines the results of the hybrid method)

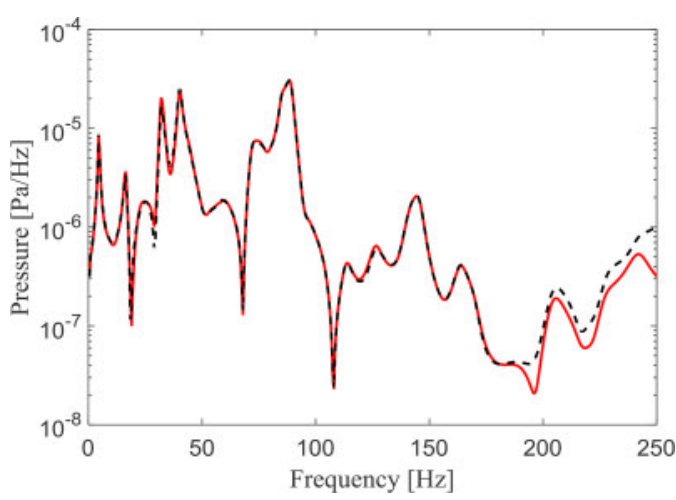

Figure 12. Frequency domain prediction of the noise pressure in center point of the upper right enclosure (Continuous red lines represent the results of the reference model and the dashed black lines the results of the hybrid method).

by both approaches for the center point of the upper right enclosure of the building. As can be seen for the frequency range up to $180 \mathrm{~Hz}$, the differences on the results are negligible for engineering purposes. 


\section{CONCLUSIONS}

This paper proposes a new approach for the prediction of railway-induced vibration and re-radiated noise in buildings to be built close to an operational railway infrastructure. The proposal is based on hybrid experimental-numerical concept. It makes use of experimental measurements of the railway-induced vibration in the ground surface to obtain the wave incident field, combined with a theoretical building/soil modelling to obtain the response of the system. This hybrid model simplifies the usual numerical procedure for these problems, since it is not required the numerical modelling of the railway infrastructure. Therefore, it reduces the uncertainty of the prediction due to the use of experimental measurements of the particular site to be studied. In addition, it provides a higher accuracy and flexibility than empirical models based on experimental transmissibility functions between the ground surface and the building. The results obtained show that the proposed hybrid model is working accurately for a $2 \mathrm{D}$ case and it can be easily generalized for $3 \mathrm{D}$ problems.

\section{ACKNOWLEDGMENTS}

This research was financially supported by: Project POCI-01-0145-FEDER-007457-CONSTRUCTInstitute of R\&D In Structures and Construction funded by FEDER funds through COMPETE2020Programa Operacional Competitividade e Internacionalização (POCI)-and by national funds through FCT-Fundação para a Ciência e a Tecnologia;Project POCI-01-0145-FEDER-029577-funded by FEDER funds through COMPETE2020 - Programa Operacional Competitividade e Internacionalização (POCI) and by national funds (PIDDAC) through FCT.

\section{REFERENCES}

[1] Quagliata, A., M. Ahearn, E. Boeker, C. Roof, L. Meister and H. Singleton, Transit Noise and Vibration Impact Assessment Manual. 2018, Federal Transit Administration-Department of Transportation, Office of Planning and Environment. Washington DC.

[2] Sadeghi, J., M.H. Esmaeili and M. Akbari, Reliability of FTA general vibration assessment model in prediction of subway induced ground borne vibrations. Soil Dynamics and Earthquake Engineering, 2019. 117: p. 300-311.

[3] Gupta, S., M.F.M. Hussein, G. Degrande, H.E.M. Hunt and D. Clouteau, A comparison of two numerical models for the prediction of vibrations from underground railway traffic. Soil Dynamics and Earthquake Engineering, 2007. 27(7): p. 608-624.

[4] Colaço, A., P. Alves Costa, P. Amado-Mendes and L. Godinho, Prediction of Vibrations and Reradiated Noise
Due to Railway Traffic: A Comprehensive Hybrid Model Based on a Finite Element Method and Method of Fundamental Solutions Approach. Journal of Vibration and Acoustics, Transactions of the ASME, 2017. 139(6).

[5] Ghangale, D., A. Colaço, P.A. Costa and R. Arcos, A Methodology Based on Structural Finite Element Method-Boundary Element Method and Acoustic Boundary Element Method Models in 2.5D for the Prediction of Reradiated Noise in Railway-Induced Ground-Borne Vibration Problems. Journal of Vibration and Acoustics, Transactions of the ASME, 2019. 141(3).

[6] Fiala, P., G. Degrande and F. Augusztinovicz, Numerical modelling of ground-borne noise and vibration in buildings due to surface rail traffic. Journal of Sound and Vibration, 2007. 301(3-5): p. 718-738.

[7] Lopes, P., P. Alves Costa, M. Ferraz, R. Calçada and A. Silva Cardoso, Numerical modeling of vibrations induced by railway traffic in tunnels: From the source to the nearby buildings. Soil Dynamics and Earthquake Engineering, 2014. 61-62: p. 269-285.

[8] Lopes, P., P. Alves Costa, R. Calçada and A. Silva Cardoso, Influence of soil stiffness on vibrations inside buildings due to railway traffic: numerical study. Computers \& Geotechnics, 2014. 61: p. 277-291.

[9] Papadopoulos, M., S. François, G. Degrande and G. Lombaert, The influence of uncertain local subsoil conditions on the response of buildings to ground vibration. Journal of Sound and Vibration, 2018. 418: p. 200-220.

[10] Auersch, L., Building Response due to Ground Vibration - Simple Prediction Model Based on Experience with Detailed Models and Measurements. Vol. 15. 2010. 101-112.

[11] Sanayei, M., A. Kayiparambil P, J.A. Moore and C.R. Brett, Measurement and prediction of train-induced vibrations in a full-scale building. Engineering Structures, 2014. 77(0): p. 119-128.

[12] López-Mendoza, D., A. Romero, D.P. Connolly and P. Galvín, Scoping assessment of building vibration induced by railway traffic. Soil Dynamics and Earthquake Engineering, 2017. 93: p. 147-161.

[13] Colaço, A., P. Alves Costa, P. Amado-Mendes, F. Magalhães and L. Godinho, Experimental validation of a FEM-MFS hybrid numerical approach for vibroacoustic prediction. Applied Acoustics, 2018. 141: p. 79-92.

[14] Arcos, R., A. Clot and J. Romeu, Dynamic representation of excitation sources on construction-induced vibration problems based on multiple harmonic loads applied on the ground. INTER-NOISE and NOISECON Congress and Conference Proceedings, 2017. 255(2): p. 5767-5774.

[15] Amado-Mendes, P., P. Alves Costa, L.M.C. Godinho and P. Lopes, 2.5D MFS-FEM model for the prediction of vibrations due to underground railway traffic. Engineering Structures, 2015. 104: p. 141-154.

[16] Alves Costa, P., R. Calçada and A. Silva Cardoso, Trackground vibrations induced by railway traffic: In-situ measurements and validation of a 2.5D FEM-BEM model. Soil Dynamics and Earthquake Engineering, 2012. 32(1): p. 111-128. 